\section{WESTINGHOUSE SAVANNAH RIVER COMPANY SAVANNAH RIVER LABORATORY DIVISION}

April 9, 1990

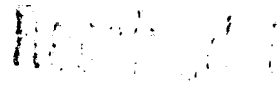

DEC $O 31990$

To: C.B. Shedrow, WSRC/EPS, 773-42A

FROM: W.L. Marter, WSRC/SRL, 773-42A

L.R. Bauer, WSRC/SRL, 773-A $L R$ bamen

\title{
DEFENSE WASTE PROCESSING FACILITY (DWPF) ENVIRONMENTAL DOSIMETRY DATA (U)
}

The original Environmental Impact Statement for the DWPF was issued in 1982. Since that time, estimated releases of radioactivity to the environment have changed because of changes in the DWPF process. In addition, the methodology for calculating offsite doses from routine releases has changed. In anticipation of a potential supplement to the $1982 \mathrm{EIS}$, current dosimetry methodology has been used to estimate offsite doses from the current as-constructed estimate of radioactivity releases. Offsite doses have also been calculated for the radioactivity release data published in the 1982 EIS using current dosimetry methodology. The two data sets may therefore be used to compare the estimated original and current impacts.

This memorandum documents the results of the offsite dose calculations for routine operation of the DWPF. Also included is a brief description of methodology and parameters used in the calculations.

Questions or comments about the enclosures may be directed to W.L. Marter (803725-5205) or to L.R. Bauer (803-725-3280).

\section{DISTRIBUTION:}

\section{WSRC}

1-10. C.B. Shedrow, $773-42$ A

11. H.D. Harmon, 773-A

12. A.L. Boni, 773-A

13. D.B. Moore, 773-A

14. D.E. Gordon, 703-52A

15. L.R. Bauer, 773-A
WSRC/DWPF SEIS Task Force

16. K.Z. Wolf, Chair, 703-H

17. W.L. Marter, 773-42A

18. J.S. Haselow, 773-42A

19. J.B. Gladden, 773-42A

20. M.D. Boersma, 773-42A 


\section{DWPF POTENTIAL SEIS ENVIRONMENTAL DOSIMETRY DATA SPECIFICATIONS}

\section{Source Terms}

Dose estimates for the 1982 Record of Decision alternative were based on atmospheric and liquid release limits reported in the Final EIS for "Stage 1, Stage 2 coupled operation" (DOE 1982). Current ("1990") atmospheric source terms were based on FY 1988 reactor operational parameters and a radionuclide blend of 5and 15-year-out-of-the-reactor waste for the insoluble and soluble fractions, respectively. This approach provides the best approximation of the waste the DWPF will process and is consistent with the NESHAPS permit application for the facility. The 1990 source terms do not include liquid effluent release rates because no direct-to-stream discharges from the DWPF are anticipated.

\section{Dosimetry Methodology}

Offsite radiological impacts for both generations of source terms were calculated using current i.e., ICRP 30 methodology. The codes used to generate wie dose estimates, and the site- and release-specific data used as input to the codes, are identified in the attachments. A more detailed description of the operational characteristics of the dose codes can be found in Reference Du Pont 1986.

\section{Description of Attachments}

1. Identification of changes in code input between FEIS and SEIS

2. Summary of doses for 1982 and 1990 source terms

3. 1990 Release Locations

4. 1990 Source Terms - H Area

5. 1990 Source Terms - S Area

6. 1990 Source Terms - Z Area

7. Dose Data - 1990 Atmospheric releases

8. 1982 Release Locations

9. 1982 Source Terms - Atmospheric releases

10. 1982 Source Terms - Liquid releases

11. Dose Data - 1982 Atmospheric releases

12. Dose Data - 1982 Liquid releases

l?: "ared by:

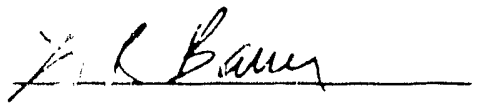

L.R. Bawer, W'SRC/SRL
Technical Review by:

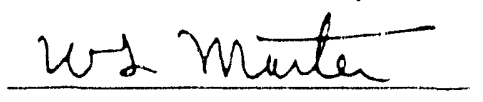

W.L. Marter, WSRC/SRI. 


\section{REFERENCES}

DOE 1982. Final Environmental Impact Statement Defense Waste Processing Facility Savannah River Plant Aiken, SC, U.S. Department of Energy, Washington, D.C., DOE/EIS-0082, February 1982.

DOE 1988a. External Dose-Rate Conversion Factors for Calculation of Dose to the Public, U.S. Department of Energy, Washington, D.C., DOE/EH-0070, July 1988.

DOE 1988b. Internal Dose Conversion Factors for Calculation of Dose to the Public, U.S. Department of Energy, Washington, D.C., DOE/EH-0071, July 1988.

Du Pont 1981a. Environmental Information Document Defense Waste Processing Facility, E.I. du Pont de Nemours \& Co., Savannah River Laboratory, Aiken, SC, DPST-80-249, July 1981.

Du Pont 1981b. Environmental Information Document Defense Waste Processing Facility Staged Operations, E.I. du Pont de Nemours \& Co., Savannah River Laboratory, Aiken, SC, DPST-80-249-Supplement, November 1981.

Du Pont 1986. Technical Manual Environmental Risk Assessment, E.I. du Pont de Nemours \& Co., Savannah River Laboratory, Aiken, SC, DPSTM-86-700-1, April 1986.

ORNL 1986. A Methodology for Calculating Radiation Doses from Radioac ivity Released to the Environment, Oak Ridge National Laboratory, Oak Ridge, TN, ORNL-4992, March 1976.

WSRC 1989. Environmental Information Document Reactor Operation Volume III. Westinghouse Savannah River Company, Savannah River Laboratory, Aiken, SC, WSRC-RP-89-817, December 1989.

\section{DISCLAIMER}

\footnotetext{
This report wis prepared as an account of work sponsored by an agency of the linted Slates Government Nelther the linted states (jovernment nor any agency therent, nur any of them

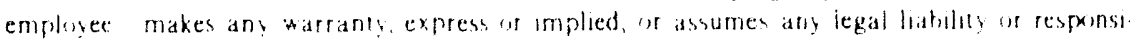
blit for the accuracy completeness. of usefuiness of any information, apparatus, prextuct, or

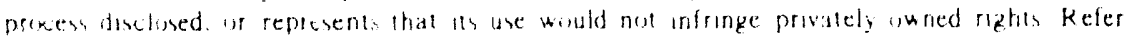

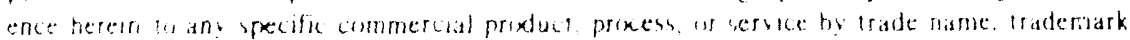

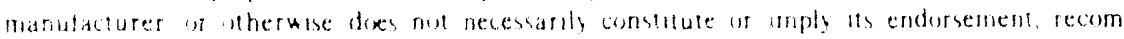

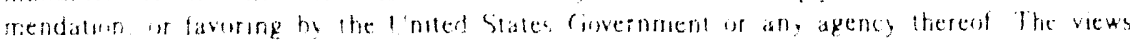

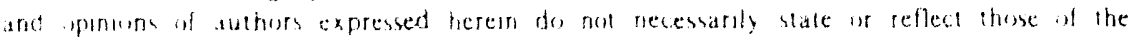
tinled slates (jovernment or ans agenc) therest
} 
ATTACHMENTS 


\section{DWPF ENVIRONMENTAL DOSIMETRY \\ Code Specifications \\ Site and Population Characteristics}

\section{Atmospheric Releases}

Dose factors

Dose codes

Maximum individual

Offsite population

80-km Population

$\%$ Adults

$\%$ Teens

$\%$ Children

\section{Liquid Releases}

Dose factors

Dose codes

Maximum inciividual

At-risk populations

River flow rate, avg cfs

River dilution in estuary

Transit time, hr

DWPF to river

SRS to WTPs

Water treatment time, hr

Aquatic food harvest, $\mathrm{kg} / \mathrm{yr}$

Fish - sport

Fish - commercial

Salt water invertebrates

Shore width fartor

Population sizes

Beaufort-Jasper

Port Wentworth

$80-\mathrm{km}$ radius

Age distribution, A/T/C

Beaufort-Jasper

Port Wentworth

$80-\mathrm{km}$ radius
1982 Data (a)

1990 Changes (b)

ICRP 2

DOE/ICRP 30

AIRDOS-EPA

AIRDOS-EPA

MAXIGASP

POPGASP

781,000

67.9

10.8

21.3

852,000

100

ICRP 2

DOE/ICRP 30

ORNL 1986

ORNL 1986

LADTAP II

LADTAP II

Factor of 3

24

72

24

90,700

31,800

299,000

0.2

40,300

29,200

117,000

781,000

852,000

$69 / 10 / 21$

$100 / 0 / 0$

$68 / 11 / 21$

$100 / 0 / 0$

$100 / 0 / 0$

$100 / 0 / 0$

(a) Based on DPST-80-249 projected averages for years 1990-2020.

(b) Based on projected values for the Year 2000 (developed for the Continued Reactor Operation EID). 


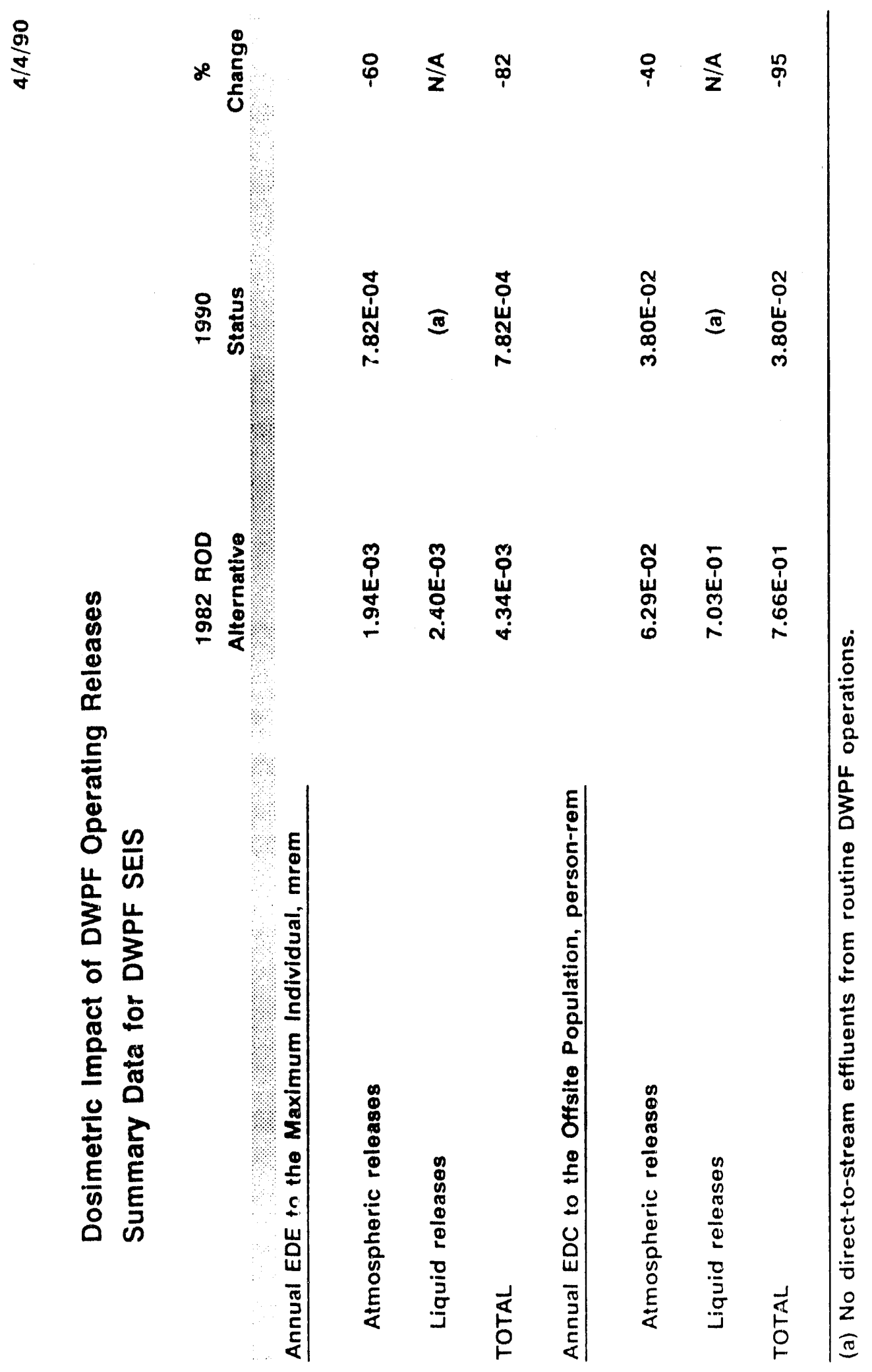


SRS

North
DWPF Release Locations 1990 Status
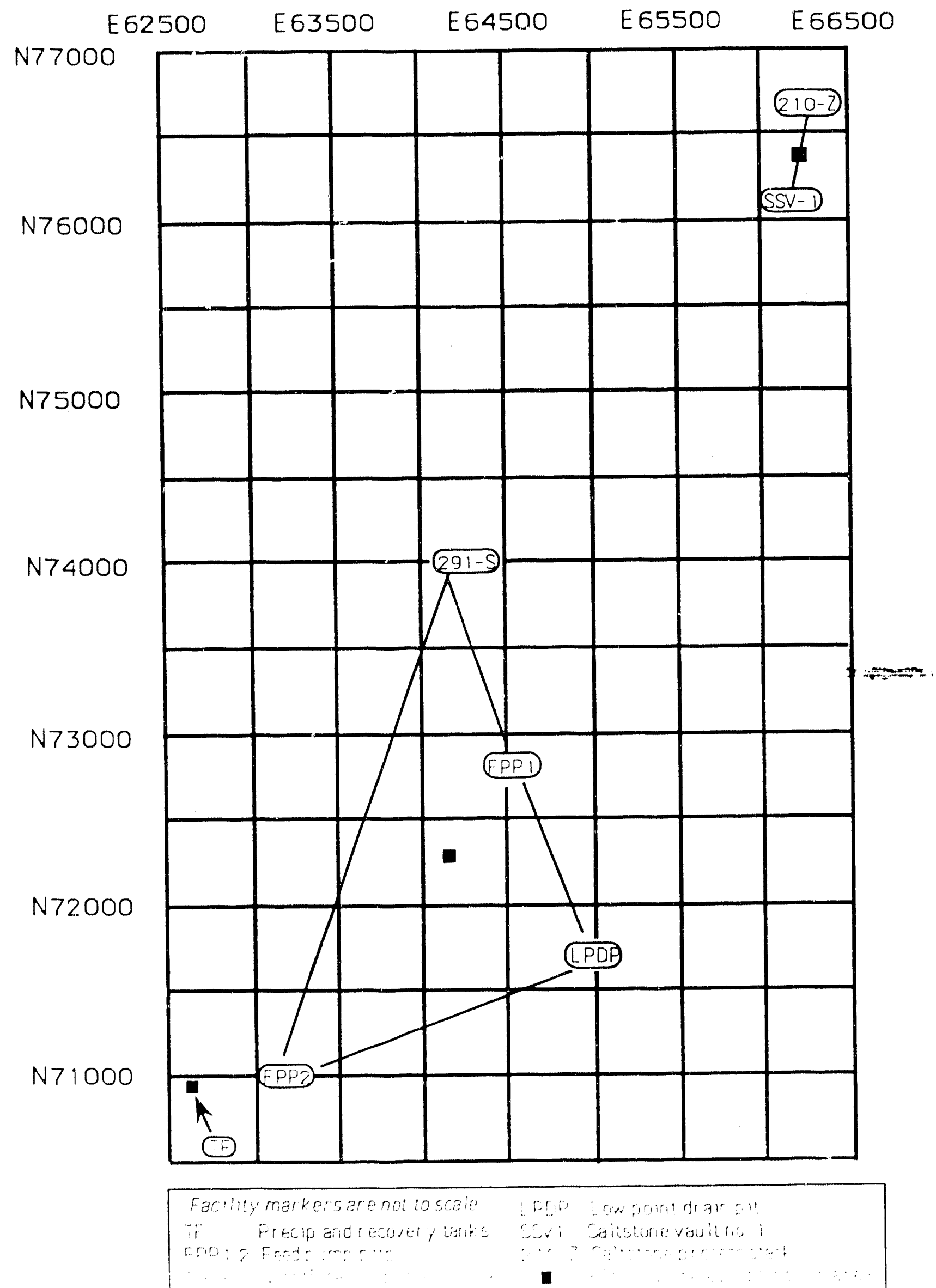


\begin{tabular}{|c|c|c|c|c|}
\hline \multicolumn{3}{|c|}{ DWPF OPERATING RELEASES - 1990 ESYIMATES } & \multicolumn{2}{|r|}{$3 / 22 / 90$} \\
\hline \multicolumn{5}{|c|}{ Annual Atmospheric $\mathrm{H}$-Area Releases, $\mathrm{Cl}$} \\
\hline & Tanks & Tank & & H-Area \\
\hline & $48 \& 49$ & 50 & & Total \\
\hline Stack Ht. (tt) & 25 & 25 & & 25 \\
\hline Coord-E & 62670 & 62605 & & 62640 \\
\hline Coord-N & 70960 & 70820 & & 70890 \\
\hline \multicolumn{5}{|l|}{ Nuclide } \\
\hline $\mathrm{H}-3$ & $1.70 E+\infty$ & $1.36 E+\infty$ & & $3.06 E+\infty$ \\
\hline C. 14 & $3.56 E-14$ & $6.81 E-13$ & & $7.17 E-13$ \\
\hline Co-60 & $5.90 E-\infty 9$ & $2.04 E-11$ & & $5.92 E-09$ \\
\hline $\mathrm{Se}-79$ & 6.75E-12 & $3.41 E-11$ & & $4.09 E-11$ \\
\hline Sr-90 & $7.18 E-06$ & $6.81 E-11$ & & $7.18 E-06$ \\
\hline Y.90 & 7.42E-06 & $6.81 E-11$ & & $7.42 E-06$ \\
\hline TC-99 & $1.25 E-00$ & $3.41 E-\infty 9$ & & $4.66 E-\infty 9$ \\
\hline$R u-106$ & $5.65 E-10$ & $3.41 E-\infty 9$ & & $3.98 E-09$ \\
\hline Rh-106 & 5.67E-10 & $3.41 E-09$ & & $3.98 E-09$ \\
\hline Sn-126 & $4.14 E-09$ & $1.36 \mathrm{E}-11$ & & $4.15 E-09$ \\
\hline Sb-125 & 2.70E-07 & $6.81 E-10$ & $\cdot$ & 2.71E-07 \\
\hline Sb-126m & $4.14 E-0.9$ & $1.36 E-11$ & & $4.15 E-09$ \\
\hline Te-125m & $3.87 E-12$ & $2.04 E-11$ & & $2.43 E-11$ \\
\hline |-129 & $2.72 E-15$ & $2.04 E-11$ & & $2.04 E-11$ \\
\hline Cs-134 & $2.99 E-06$ & $6.81 E-12$ & & $2.99 \mathrm{E}-06$ \\
\hline Cs-135 & $1.51 \mathrm{E}-09$ & $4.08 E-15$ & & 1.51E-09 \\
\hline$C s-137$ & $6.48 E-04$ & $2.04 E-09$ & & 6ABEOL \\
\hline Ba-137m & $6.19 E-04$ & $2.04 E-09$ & & $6.19 E-04$ \\
\hline Ce-144 & $9.20 E-11$ & $3.41 E-13$ & & $9.23 \mathrm{E}-11$ \\
\hline Pr-144 & $9.23 E-11$ & $3.41 E-13$ & & $9.26 E-11$ \\
\hline Fr-144m & $1.10 E-12$ & $3.41 E-15$ & & $1.10 E-12$ \\
\hline Pm-147 & $1.18 E-07$ & $4.09 E-10$ & & $1.18 E-07$ \\
\hline Sm-151 & $5.44 E-08$ & $2.04 E-10$ & & $5.46 E-08$ \\
\hline$E u-152$ & $2.11 E-10$ & $6.13 E \cdot 13$ & & $2.12 E-10$ \\
\hline Eu-154 & $2.47 E-08$ & $6.81 E-11$ & & $2.48 E-08$ \\
\hline Eu-155 & $9.97 E-\infty 9$ & $3.41 E-11$ & & $1.00 E-08$ \\
\hline$P u-238$ & 2.75E-07 & 4.77E-12 & & 2.75E-07 \\
\hline Pu-239 & $2.50 E-09$ & $4.77 E-14$ & & $2.59 E-09$ \\
\hline$P u-240$ & $1.75 E-\infty 9$ & $3.41 E-14$ & & $\therefore .75 E-09$ \\
\hline Pu-241 & $2.09 E-07$ & $3.41 \mathrm{E}-12$ & & $2.09 E-07$ \\
\hline Am-241 & $4.32 E-09$ & $1.36 \mathrm{E}-11$ & & 4.33E- 09 \\
\hline $\mathrm{Cm}-244$ & $2.16 \mathrm{E}-08$ & $6.81 E-14$ & & 2.16E.08 \\
\hline
\end{tabular}




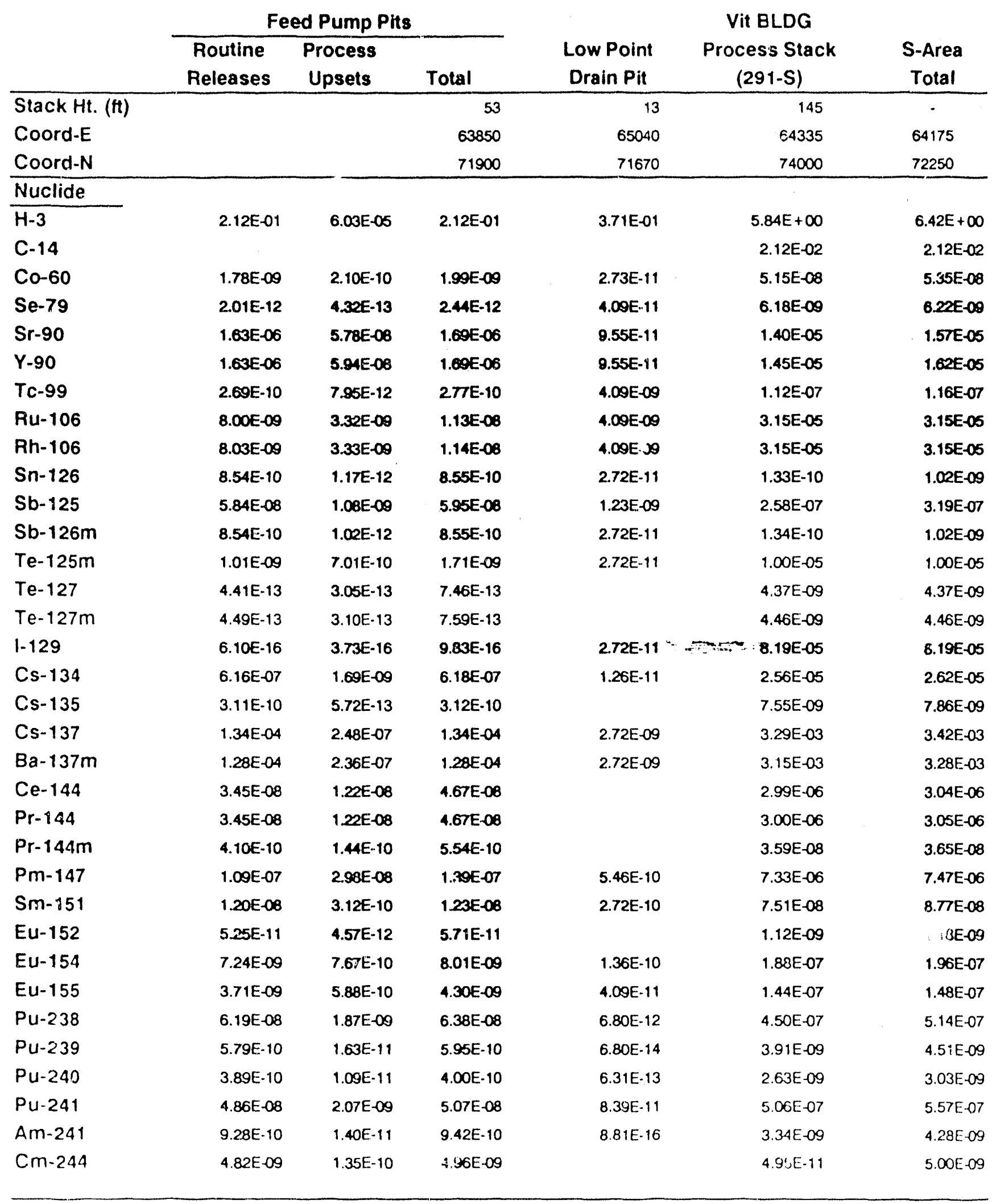


DWPF OPERATING RELEASES - 1990 ESTIMATES

$3 / 22 / 90$

Annual Atmospheric Z-Area Releases, $\mathrm{CI}$

\begin{tabular}{|c|c|c|c|}
\hline & $\begin{array}{c}\text { Saltstone } \\
\text { Process Stack } \\
(210-Z) \\
\end{array}$ & $\begin{array}{c}\text { Saltstone } \\
\text { Vault } \\
\text { No. } 1 \\
\end{array}$ & $\begin{array}{c}\text { Z-Area } \\
\text { Total }\end{array}$ \\
\hline Stack Ht. (ft) & 41 & 15 & GL (a) \\
\hline Coord-E & 66310 & 66200 & 66255 \\
\hline Coord-N & 76620 & 76052 & 76340 \\
\hline \multicolumn{4}{|l|}{ Nuclide } \\
\hline $\mathrm{H}-3$ & 4.60E-01 & $2.10 E+01$ & $2.15 E+01$ \\
\hline Co-60 & $1.73 E-\infty \theta$ & & $1.73 E-09$ \\
\hline Se-79 & $258 E-00$ & & $2.58 E-\infty 9$ \\
\hline Sr-90 & $6.04 E-\infty 0$ & & $6.04 E-09$ \\
\hline Y-90 & $6.04 E-\infty 9$ & & $6.04 E-09$ \\
\hline TC-99 & $258 E-07$ & & 2.58E-07 \\
\hline$R u-106$ & $258 E-07$ & & $2.585=-07$ \\
\hline Rh-106 & $258 E-07$ & & $2.58 E-07$ \\
\hline Sn-126 & $1.72 E-09$ & & $1.72 E-09$ \\
\hline$S b-125$ & $7.75 E-08$ & & $7.75 E-08$ \\
\hline$S b-126 m$ & $1.72 E-09$ & & $1.72 E-09$ \\
\hline Te-125m & $1.72 E-09$ & & $1.72 E-09$ \\
\hline $1-129$ & $1.72 E-09$ & & $1.72 E-09$ \\
\hline$C_{8-134}$ & $7.976-10,0$ & & 7.97E-10 \\
\hline Cs-137 & $1.72 E-07$ & & $1.72 E-07$ \\
\hline $\mathrm{Ba}-137 \mathrm{~m}$ & $1.72 E-07$ & & $1.72 E-07$ \\
\hline Pm-147 & $3.44 E-08$ & & 3.44E- -08 \\
\hline Sm-151 & $1.72 E-08$ & & $1.72 .5-08$ \\
\hline Eu-154 & $8.62 E-09$ & & 8.62E-09 \\
\hline Eu-155 & $2.58 \mathrm{E}-09$ & & $2.58 E-09$ \\
\hline Pu-238 & 4.31E-10 & & 4.31E-10 \\
\hline Pu-239 & $4.31 E-12$ & & 4.31E-12 \\
\hline Pu-240 & $3.99 E-11$ & & 3.99E-11 \\
\hline Pu-241 & $5.30 E-09$ & & 5.30E- -09 \\
\hline Am-241 & $5.56 E-14$ & & $5.56 \mathrm{E}-14$ \\
\hline
\end{tabular}

(a) $G L=$ Treated as a yround tevel release. 


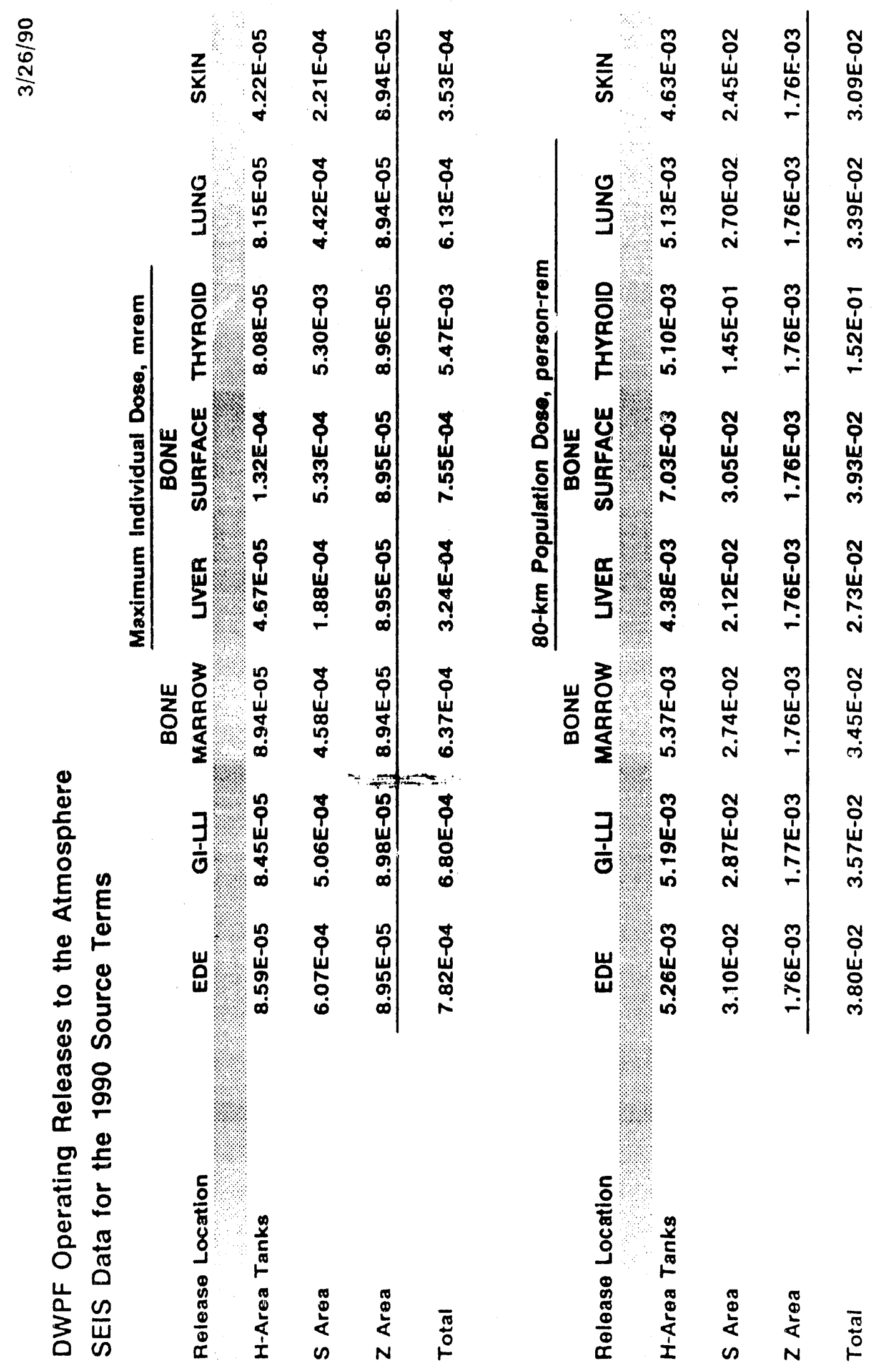


$\triangle$ SRS

North
DWPF Releose Locations

1982 Stotus

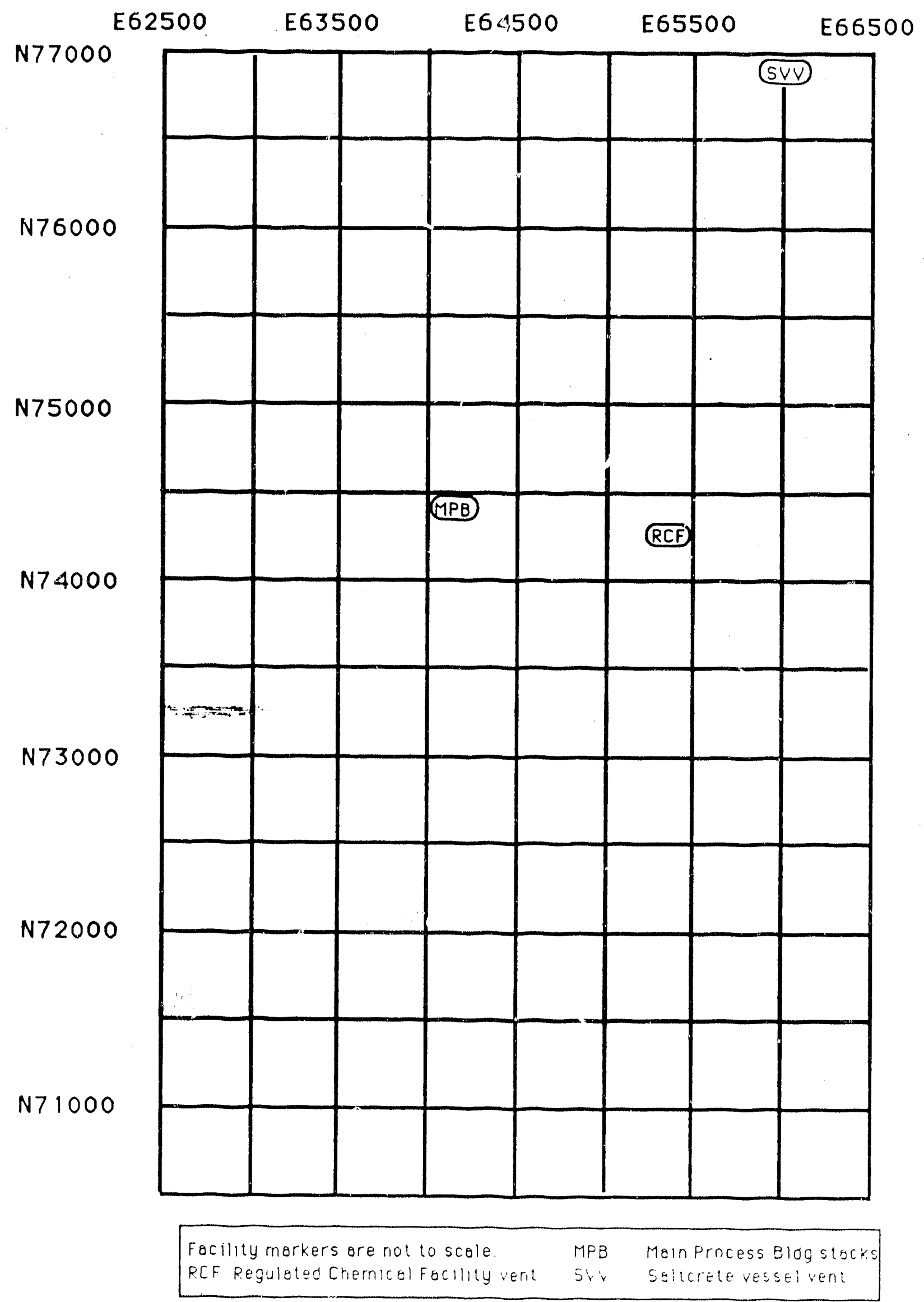


DWPF OPERATING RELEASES - FINAL EIS (FEB 1982)

Annual Atmospheric Releases, $\mathrm{Cl}$

Stage 1 / Stage 2 Coupled Atternative (a)

\begin{tabular}{|c|c|c|c|c|}
\hline & $\begin{array}{c}\text { Sand Filter } \\
\text { Stack }\end{array}$ & $\begin{array}{c}\text { Regulated } \\
\text { Chemical Facility }\end{array}$ & $\begin{array}{c}\text { Saltcrete } \\
\text { Plant } \\
\end{array}$ & Total \\
\hline Stack Ht. (ft) & 140 & Ground Level & Ground Level & $\cdot$ \\
\hline Cisord-E & 64020 & 65395 & 66000 & - \\
\hline Coord-N & 74440 & 74270 & 76295 & - \\
\hline \multicolumn{5}{|l|}{ Nuclide } \\
\hline$H-3$ & $5.4 E+\infty 0$ & $2.3 E+00$ & $2.3 E+00$ & $1.0 E+01$ \\
\hline Co-60 & $1.2 E \times 05$ & 2.7E-10 & $3.5 E-10$ & 1.2E-05 \\
\hline Sr-90 & $2.1 E-13$ & $3.5 E-10$ & $3.8 E-10$ & $2.1 E-03$ \\
\hline$Y-90$ & 2.1E-03 & $3.5 E-10$ & $3.8 \mathrm{E}-10$ & $4.2 E-03$ \\
\hline TC-99 & 3.6E-07 & $1.4 E-08$ & $1.9 E-08$ & $3.9 E-07$ \\
\hline Ru-106 & $3.0 E-04$ & $9 . \approx E-09$ & $1.2 E-08$ & $3.0 E-04$ \\
\hline Rh-106 & $3.0 E-04$ & $9.2 E-09$ & $1.2 E-08$ & 3.0E-04 \\
\hline$S b-125$ & 5.9E-05 & 3.9E-09 & 5.1E-09 & $5.9 E-05$ \\
\hline $\mathrm{Te}-125 \mathrm{~m}$ & $2.8 E-05$ & $4.8 E-09$ & $6.2 E-09$ & 2.8E-05 \\
\hline $1-129$ & $4.2 E-04$ & $4.4 E-11$ & 5.7E-11 & 4.2E-04 \\
\hline Cs-134 & $1.0 E-05$ & $7.4 E-11$ & $9.7 E-11$ & $1.0 \mathrm{E}-05$ \\
\hline$\dot{C} s=13 ?^{-}$ & 2.1E-03 & $1.6 E-08$ & $2.0 E-08$ & 2.1E-03 \\
\hline $\mathrm{Ce}-144$ & $6.9 E-04$ & $9.3 E-11$ & $1.2 E-10$ & $6.9 E-04$ \\
\hline$P_{Y-144}$ & $6.9 E-04$ & $9.3 E-11$ & $1.2 \mathrm{E}-10$ & 6.9E-04 \\
\hline Pm-147 & $1.7 E \cdots 03$ & $1.0 E-07$ & 1.3E-07 & $1.7 E-03$ \\
\hline Sm-151 & 1.7E-05 & $1.3 E-08$ & 1.7E-08 & 1.7E-05 \\
\hline Eu-154 & 4.4E-05 & $1,6 E-09$ & 2.1E-09 & 4.4E-05 \\
\hline Eu-155 & 3.5E-05 & $6.8 E-10$ & $9.0 \mathrm{E}-10$ & $3.5 E-05$ \\
\hline Pu-238 & $5.3 E-05$ & $2.6 E-11$ & $3.4 E-11$ & $5.3 E-05$ \\
\hline Pu-241 & 5.9E-05 & $2.0 E-11$ & $2.6 \mathrm{E}-11$ & $5.9 E-05$ \\
\hline
\end{tabular}

(a) Location and source term data based on DPST -80-249 (Jul 1981), a supplement to that EID (Nov 1982), and the Final EIS DOE/EIS-0082 (Fob 1982). 
DWPF OPERATING RELEASES - FINAL EIS (FEB 1982)

Annual Liquid Releases, $\mathrm{Cl}$

Stage 1 / Stage 2 Coupled Alternative

\begin{tabular}{lc} 
Nuclide & $\begin{array}{r}\text { Waste Tank Farm Evapurator } \\
\text { Releases to the Savannah River }\end{array}$ \\
\hline H-3 & $8.5 E+02$ \\
Sr-90 & $2.3 E-05$ \\
Y-90 & $2.3 E-05$ \\
Tc-99 & $4.6 E-09$ \\
Ru-106 & $3.0 E-09$ \\
Rh-106 & $3.0 E-09$ \\
Sb-125 & $1.3 E-09$ \\
Te-125m & $1.5 E-09$ \\
I-129 & $1.4 E-11$ \\
Cs-134 & $2.4 E-11$ \\
Cs-137 & $5.1 E-09$ \\
Ea-137m & $4.8 E-09$ \\
Ce-144 & $3.0 E-11$ \\
Pr-144 & $3.0 E-11$ \\
Pm-147 & $3.3 E-08$ \\
Sm-151 & $4.1 E-09$ \\
Max discharge rate $=11 \mathrm{~L} / \mathrm{min}$. & \\
\hline
\end{tabular}


$\frac{\stackrel{\circ}{9}}{\frac{m}{m}}$

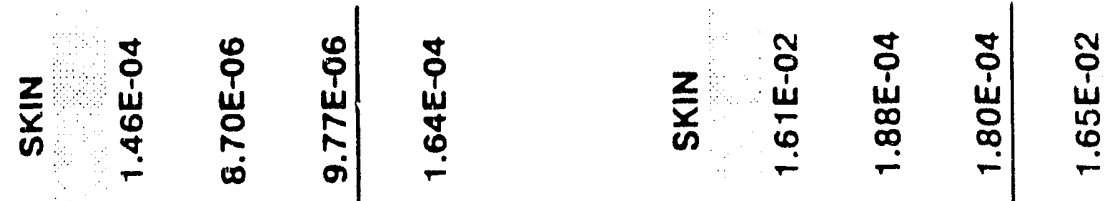

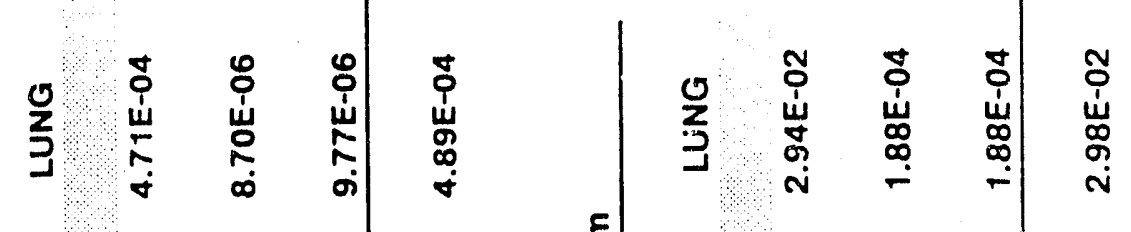

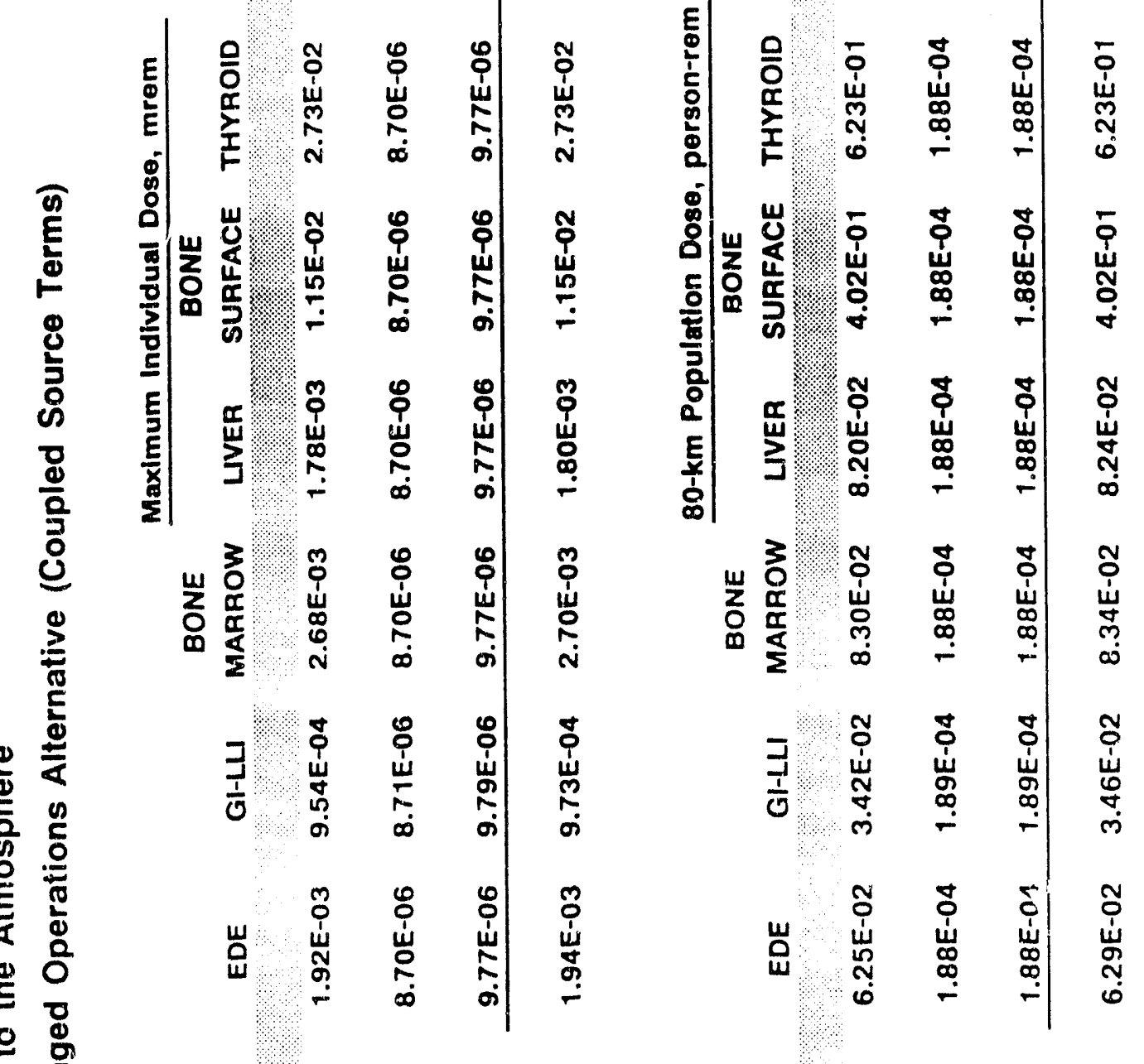

\& क

袮

g)

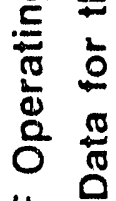

耑竞 $\frac{0}{\omega}$

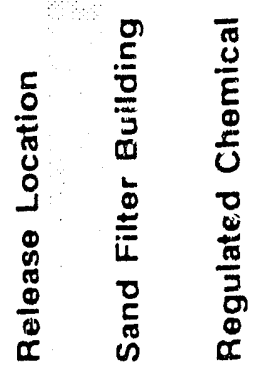

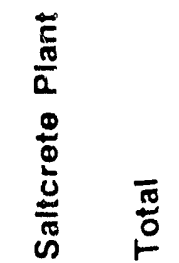

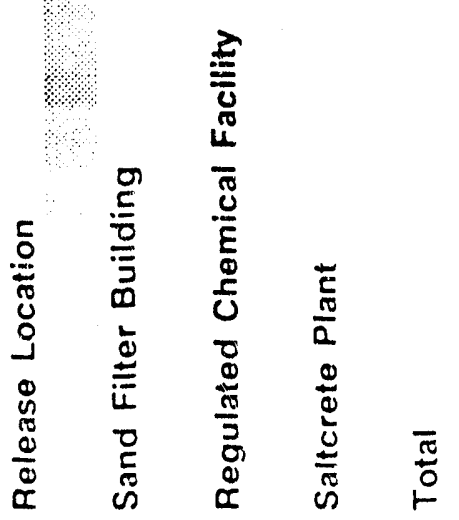


$\frac{8}{\frac{m}{m}}$

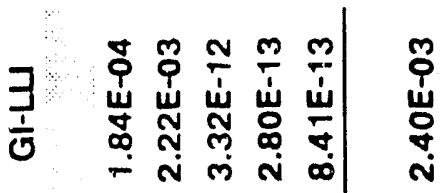

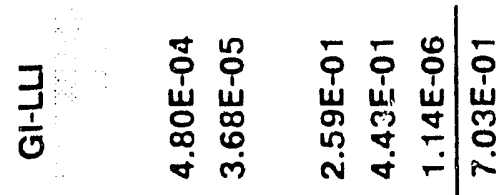

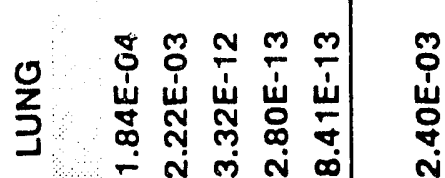

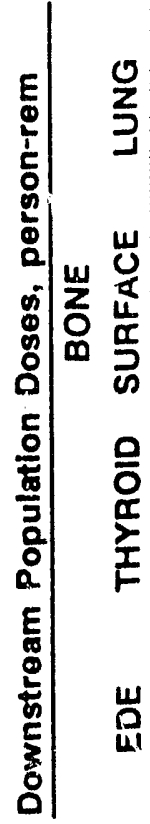

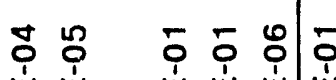

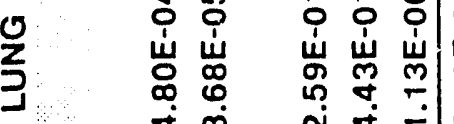

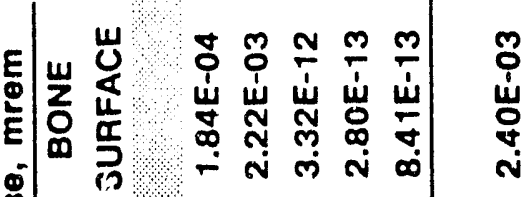

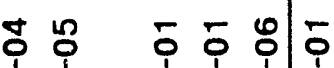

岁 岁岕岗

宁

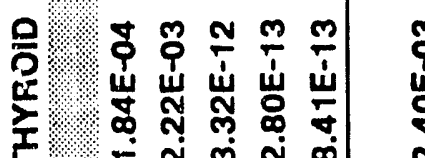

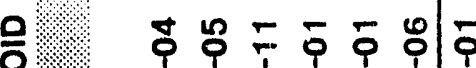

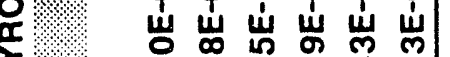

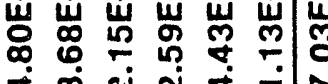

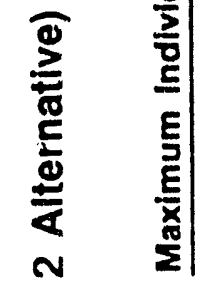

\& $\cong \dddot{9} \div$

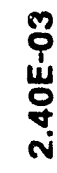

吉 n

㟔?

a)

品

䍃 岀 嵌 㟧

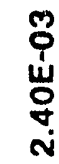

妥

㟧㟧峲岕岁 我兽

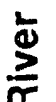

寸

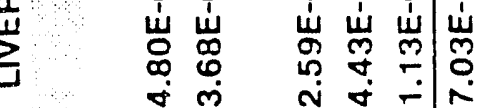

ㄷํำ

禹

\&

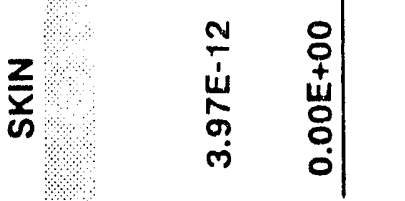

$\underset{\substack{\text { L } \\ \stackrel{\alpha}{\sigma}}}{\stackrel{\sim}{r}}$

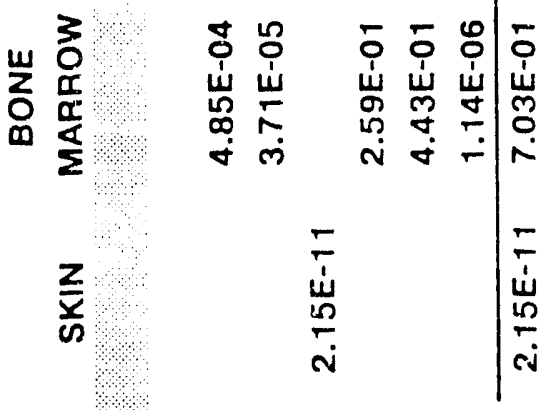

\&

운

边

岕

ז.

㡙

过

号

嵅 $\frac{\omega}{w}$
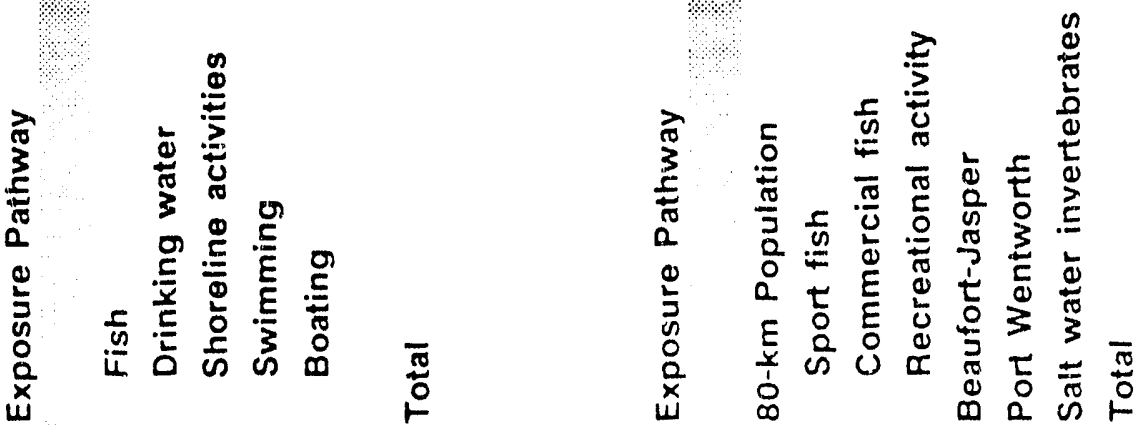

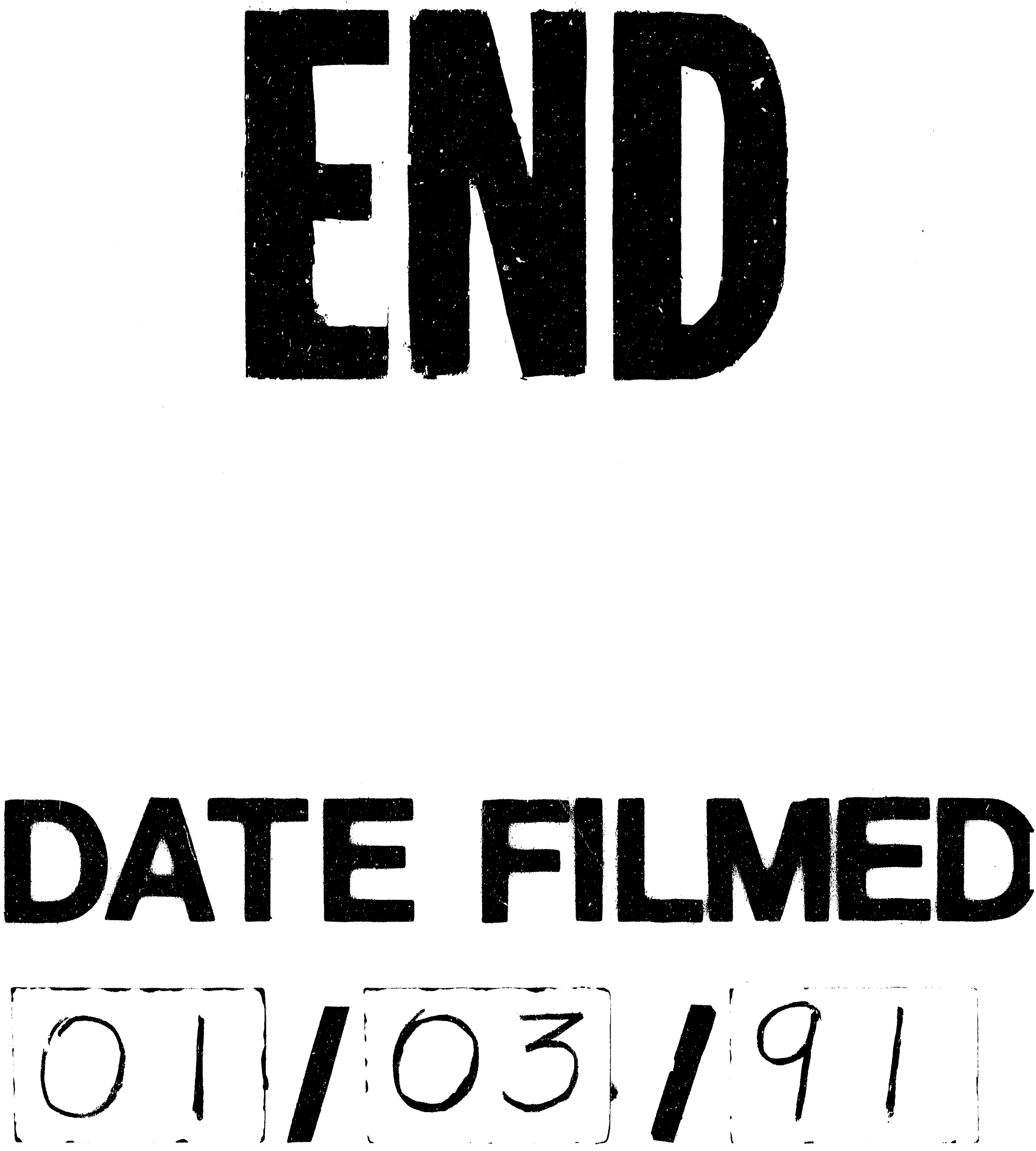


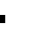

